\title{
Diabetic Ketoacidosis and Acute Mountain Sickness: Case Report and Review of Treatment Options in Type 1 Diabetes Mellitus
}

\author{
Steven C.M. Miller, BSc, MBChB, PhD, FRCP, FRACP \\ From the Department of Endocrinology, North Shore Hospital, Takapuna, Auckland, New Zealand.
}

\begin{abstract}
A 30-year-old man with a 20-year history of well-controlled type 1 diabetes mellitus and no microvascular complications traveled from near sea level to an altitude of $3000 \mathrm{~m}$ within 6 hours. At altitude, his blood glucose levels began to rise, necessitating increased insulin delivery. Typical symptoms of acute mountain sickness (AMS) developed, and he became increasingly hyperglycemic and unwell. Upon presentation to an emergency clinic, diabetic ketoacidosis (DKA) was diagnosed and was managed with insulin, intravenous fluids with potassium, and acetazolamide orally. No other potential causes for diabetic ketoacidosis were identified. Hyperglycemia, ketosis, and acidosis resolved with treatment as expected, but an increased insulin requirement was noted for the next 48 hours, until returning to expected levels when acetazolamide was discontinued. This case describes an episode of mild diabetic ketoacidosis potentially precipitated by moderate to severe acute mountain sickness, and an apparent hyperglycemic effect of acetazolamide. Individuals with type 1 diabetes traveling to altitude and their physicians should be vigilant for this complication and should be aware of the effects of conventional first-line therapies for acute mountain sickness on insulin requirement, glycemic control, and preexisting microvascular diabetes complications.
\end{abstract}

Key words: diabetic ketoacidosis, DKA, acute mountain sickness, AMS, type 1 diabetes mellitus, T1DM, acetazolamide, dexamethasone

\section{Introduction}

Unacclimatized individuals who travel to altitudes of more than $2500 \mathrm{~m}$ in a single day are at risk of having acute mountain sickness (AMS). ${ }^{1}$ Descent of $300 \mathrm{~m}$ to $1000 \mathrm{~m}$ is considered to be the single best treatment for AMS; however, treatment options such as supplemental oxygen therapy, the carbonic anhydrase inhibitor acetazolamide, or the synthetic glucocorticoid dexamethasone (both administered orally) are more usually used as first-line treatment. Individuals with type 1 diabetes mellitus require exogenous insulin, delivered either by multiple daily subcutaneous injections or continuous subcutaneous infusion through an insulin pump. The insulin dose or delivery rate is adjusted to maintain blood glucose at near-normal levels. ${ }^{2}$ An increased requirement for insulin with increasing altitude is recognized among some individuals with type 1 diabetes. $^{3}$

Corresponding author: Steven C.M. Miller, PhD, Department of Endocrinology, North Shore Hospital, Shakespeare Road, Takapuna, Auckland 0629, New Zealand (e-mail: steven@endocrinology.co.nz).
Diabetic ketoacidosis (DKA) is a life-threatening complication of type 1 diabetes that occurs when insufficient insulin is delivered, requiring prompt administration of insulin, fluids, and electrolytes to restore normal metabolism. ${ }^{4}$ There are few reports of DKA due to AMS in the literature, possibly reflecting the reluctance of those with diabetes to travel to such locations. Recent advances that facilitate optimized glycemic self-management in type 1 diabetes such as insulin pumps and continuous glucose monitors (reviewed by DeSalvo and Buckingham ${ }^{5}$ ) have led to an increasing number of individuals with type 1 diabetes traveling to altitude, often in remote locations. A thorough understanding of the optimum treatment of DKA due to AMS is, therefore, an essential goal. Metabolic decompensation (hyperglycemia) and increased insulin requirement is almost universally observed when supraphysiological doses of glucocorticoids are administered to patients with type 1 diabetes. ${ }^{6}$ As there have been no randomized, placebocontrolled studies, the effects of acetazolamide on insulin 
requirement and glycemic control of type 1 diabetes is less clear.

Here, I describe a case of DKA potentially due to severe AMS precipitated by rapid ascent to moderate altitude, and the subsequent effects of treatment with acetazolamide on insulin requirement. The potential adverse effects of conventional treatments for AMS are reviewed. On account of these potential adverse effects, alongside the morbidity and mortality associated with DKA, descent from altitude may be a safer alternative when DKA is precipitated by AMS in individuals with type 1 diabetes.

\section{Case Presentation}

A 30-year-old man traveled from his home in Los Angeles, California (elevation $236 \mathrm{~m}[774 \mathrm{ft}]$ ), to Breckenridge, Colorado (elevation 2926 m [9600 ft]), above sea level over the course of 6 hours with no acclimatization. He had been diagnosed with type 1 diabetes at the age of 8 years and had maintained good glycemic control (hemoglobin A1C 48 to $53 \mathrm{mmol} / \mathrm{mol}$ [6.5\% to $7 \%$ ] over the past 12 months). In the 21 years he had type 1 diabetes, he had 3 hospital admissions with severe hypoglycemia (last episode 18 months earlier) and 3 previous episodes of DKA (last episode 3 years earlier), all with a clear precipitant. There was no evidence of microvascular or macrovascular complications. Insulin (lispro) was delivered via an insulin pump, and glycemia monitored using a continuous glucose monitor. Regular adjustments of insulin delivery allowed maintenance of blood glucose within a narrow range. He reported no symptoms suggestive of intercurrent illness during the week before travel and the week after this episode. Comorbidities included mild hypertension (treated with amlodipine, $2.5 \mathrm{mg}$ daily) and posttraumatic stress disorder (treated with sertraline, $25 \mathrm{mg}$ daily). He denied the use of any over-the-counter medication or supplements, and he had no known drug allergies. He reported no prior episodes of AMS.

Six hours after arriving at altitude, he developed mild generalized weakness, dizziness, nausea, frontal headache, and he was unable to sleep (Lake Louise Score = 9). ${ }^{7}$ Blood glucose level was persistently elevated at 240 $\mathrm{mg} / \mathrm{dL}(13.3 \mathrm{mmol} / \mathrm{L})$ in spite of an increased basal insulin infusion rate $(150 \%)$ and two 3-unit correction boluses. His symptoms worsened, vomiting ensued, and he sought medical attention.

At the local primary care medical center (elevation approximately $3000 \mathrm{~m}[9800 \mathrm{ft}]$ ), the patient's blood $\mathrm{pH}$ was acidic, whole blood glucose was elevated at $350 \mathrm{mg} /$ $\mathrm{dL}(19.5 \mathrm{mmol} / \mathrm{L})$, and capillary beta hydroxybutyrate (ketone) was $1.4 \mathrm{mmol} / \mathrm{L}$, indicating moderate ketoacidosis. Increased basal insulin infusion rate with 3 to 4 hourly correction boluses were continued. Three liters intravenous normal saline with potassium $(40 \mathrm{mmol}$ $\mathrm{KCl} / \mathrm{L}$ ) was administered over 4 hours. Intravenous antiemetic (ondansetron, $4 \mathrm{mg}$ ) was given, and oral acetazolamide, $125 \mathrm{mg}$ twice daily, was begun. Within 90 minutes of beginning treatment, his blood glucose level was $250 \mathrm{mg} / \mathrm{dL}(13.9 \mathrm{mmol} / \mathrm{L})$ and continued to fall. Ketones fell to $1.2 \mathrm{mmol} / \mathrm{L}$, indicating resolving DKA. Once the intravenous fluid was completed, the patient's symptoms were significantly improved, and he was discharged.

Acetazolamide was continued for 48 hours during which time an increased basal insulin infusion rate of $130 \%$ was required to maintain the patient's betweenmeal euglycemia, and correction doses were required to prevent postprandial hyperglycemia. Symptoms of AMS completely resolved within 24 hours of presentation. When the acetazolamide was discontinued, his blood glucose level tended to fall toward the hypoglycemic range, necessitating a reduction in the basal infusion rate back to normal levels, and no more correction boluses were needed. Basal, mealtime bolus, and total daily insulin dose, shown in Figure 1, demonstrate increases in both basal and mealtime bolus dose requirements while acetazolamide was used. The drop in insulin requirement, from $130 \%$ of normal back to baseline levels, on discontinuation of acetazolamide was abrupt.

\section{Discussion}

Acute mountain sickness is a risk when travel to altitudes higher than $2500 \mathrm{~m}$ is undertaken, and predicting who will be affected is difficult. ${ }^{1}$ A number of researchers have reported no difference in the incidence of AMS between healthy climbers and climbers with type 1

Basal, Bolus and Total Daily Insulin Dose

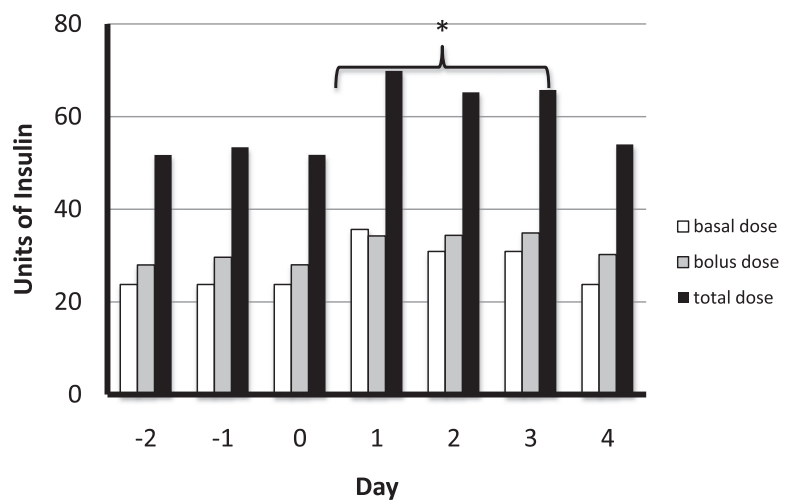

Figure 1. Total daily basal insulin dose (open bars), total daily bolus insulin dose (shaded bars), and total daily insulin dose (solid bars). Day 0 indicates travel day. *Indicates significantly increased insulin requirement on days 1 to 3 compared with other days $(P<.05)$. 
diabetes, even at altitudes above $5000 \mathrm{~m} .^{3,8-10}$ Type 1 diabetes per se, therefore, does not seem to increase AMS susceptibility; however, metabolic decompensation may occur as a result of AMS, as in the case described. The changes in insulin requirement observed at altitude for individuals with type 1 diabetes do not seem to be uniform, with conflicting reports of both increased and decreased total daily insulin requirement at various (or varying) altitudes. ${ }^{9,10}$ These reports, however, are likely to be affected by the confounding effects of increased physical activity, which is in itself likely to reduce insulin requirement. Among individuals without diabetes, fasting insulin and markers of insulin resistance, inflammation, and oxidative stress are observed to rise with the development of hypoxemia at altitude, suggesting a potential mechanism. ${ }^{11}$ Given that reduced insulin sensitivity has been suggested as a marker for AMS, ${ }^{12}$ any sudden increase in insulin requirement to maintain euglycemia in an individual with T1DM at altitude may herald the development of AMS, and should prompt heightened self-care to ensure increased insulin delivery to prevent DKA, especially in the wilderness.

Conventional treatment of DKA requires intravenous insulin, fluids, and electrolytes in addition to treating any underlying cause. In an austere environment, such treatments are unlikely to be immediately available. Indeed, DKA has been reported to be responsible for the deaths of diabetic individuals while participating in a Himalayan trek, ${ }^{13}$ and lack of access to an appropriate healthcare facility is likely to have been a contributory factor. Therefore, additional care should be taken, even more so than normal, to avoid DKA in the wilderness.

Acetazolamide is considered as both prophylaxis and first-line therapy to treat AMS, and it was given in this case. There are reasons why acetazolamide may not be appropriate therapy for AMS with type 1 diabetes. The accepted mode of action of acetazolamide in AMS is through renal carbonic anhydrase inhibition, which leads to increased ventilation (a number of noncarbonic anhydrase-mediated effects have also been proposed, ${ }^{14}$ which may be less effective in type 1 diabetes ${ }^{15}$ ). The resulting urinary loss of bicarbonate would be harmful in the setting of DKA, in which bicarbonate is an essential buffer to help protect against development of acidosisloss of bicarbonate will worsen metabolic acidosis. Acetazolamide given for AMS has previously been associated with persistent acidosis in type 1 diabetes when treatment of DKA had led to restoration of euglycemia and clearance of ketones. ${ }^{9}$ In the case described above, the use of acetazolamide was coincident with an increased insulin requirement that persisted after the resolution of DKA; the patient's insulin requirement rapidly returned to baseline when acetazolamide was withdrawn. That suggests a potential influence of acetazolamide on insulin sensitivity (and thus insulin requirement). Further study of the effects of acetazolamide on insulin requirement in type 1 diabetes are required. Until the results of such investigations are known, I suggest caution when considering acetazolamide therapy for individuals with type 1 diabetes.

Dexamethasone is a potent synthetic glucocorticoid and is accepted effective therapy for AMS. ${ }^{1}$ The ability of glucocorticoids to promote insulin resistance and even cause type 2 diabetes is well established. With type 1 diabetes, steroids also promote resistance to insulin, and increased insulin requirement to maintain euglycemia is observed. Steroids are well recognized as a causative factor for major metabolic decompensation when used as therapeutic agents in other fields of medicine. ${ }^{16}$ Diabetic ketoacidosis represents an insulin-resistant state, and resolution would be expected to be delayed if insulin requirement was increased even further by the administration of glucocorticoids. Moreover, supraphysiological glucocorticoids can lead to increased kaliuresis (by a renal mineralocorticoid effect), an undesirable outcome in an already potassium-depleted state. Hence, dexamethasone may not be appropriate therapy for AMS that has caused DKA in an individual with type 1 diabetes. I recommend that dexamethasone not be used to treat mild AMS with type 1 diabetes, reserving it for treating severe AMS or high altitude cerebral edema in individuals with type 1 diabetes.

In summary, I describe a case of AMS that may have precipitated mild DKA in a 30-year-old man with longstanding uncomplicated type 1 diabetes and, normally, good glycemic control. He was treated with acetazolamide, 125 mg twice daily, which led to an apparent increased insulin requirement of $30 \%$, which resolved immediately when acetazolamide was discontinued. The metabolic effects of acetazolamide on type 1 diabetes are largely unknown, prompting the need for further study. Dexamethasone, an alternative therapy for AMS, is likely to worsen DKA. Type 1 diabetes per se should not preclude travel to altitude. Graded ascent remains the best practice to avoid AMSand that should be emphasized for patients with type 1 diabetes-because potential metabolic complications may arise from taking the drugs conventionally used to treat the condition. Until further evidence is available to inform practice, descent from altitude may be the safest alternative to acetazolamide and dexamethasone when DKA is precipitated by AMS in individuals with type 1 diabetes.

\section{References}

1. Luks AM, McIntosh SE, Grissom CK, et al. Wilderness Medical Society consensus guidelines for the prevention 
and treatment of acute altitude illness. Wilderness Environ Med. 2010;21:146-155.

2. Standards in medical care in diabetes 2014. Diabetes Care. 2014;37(Suppl):14-80.

3. de Mol P, de Vries ST, de Koning EJ. Increased insulin requirements during exercise at very high altitude in type 1 diabetes. Diabetes Care. 2011;34:591-595.

4. Savage MW. Management of diabetic ketoacidosis. Clin Med. 2011;11:154-156.

5. DeSalvo D, Buckingham B. Continuous glucose monitoring: current use and future directions. Curr Diabetes Rep. 2013;13:657-662.

6. Yuen KC, Chong LE, Riddle MC. Influence of glucocorticoids and growth hormone on insulin sensitivity in humans. Diabet Med. 2013;30:651-663.

7. Sutton JR, Coates G, Houston CS, eds. Hypoxia and Mountain Medicine. New York, NY: Pergamon Press; 1992.

8. Leal C. Going high with type 1 diabetes. High Alt Med Biol. 2005;6:14-21.

9. Moore K, Vizzard N, Coleman C, et al. Extreme altitude mountaineering and type 1 diabetes: the Diabetes Federation of Ireland Kilimanjaro Expedition. Diabet Med. 2001;18:749-755.
10. Pavan P, Sarto P, Merlo L, et al. Extreme altitude mountaineering and type 1 diabetes. Diabetes Care. 2003;26:3196-3197.

11. Siervo M, Riley HL, Fernandez BO, et al. Effects of prolonged exposure to hypobaric hypoxia on oxidative stress, inflammation and gluco-insular regulation: the notso-sweet price for good regulation. PLoS One. 2014;9: e94915.

12. Spliethoff K, Meier D, Aeberli I, et al. Reduced insulin sensitivity as a marker for acute mountain sickness? High Alt Med Biol. 2013;14:240-250.

13. Shlim DR, Gallie J. The causes of death among trekkers in Nepal. Int J Sports Med. 1992;13:74-76.

14. Swenson E. Carbonic anhydrase inhibitors and high altitude illnesses. Subcell Biochem. 2014;75: 361-386.

15. Fülesdi B, Limburg M, Oláh L, et al. Lack of gender difference in acetazolamide-induced cerebral vasomotor reactivity in patients suffering from type- 1 diabetes mellitus. Diabetologia. 2001;38:107-112.

16. Daniel BT. Glycemic crises in patients with hematologic malignancies. Crit Care Nurs Clin North Am. 2000;12: 297-305. 